\title{
Out-of-plane magnetic patterning on austenitic stainless steels using plasma nitriding
}

\author{
E. Menéndez, ${ }^{1, a)}$ J.-C. Stinville, ${ }^{2}$ C. Tromas, ${ }^{2}$ C. Templier, ${ }^{2}$ P. Villechaise, ${ }^{2}$ J.-P. Rivière, ${ }^{2}$ \\ M. Drouet, ${ }^{2}$ A. Martinavičius, ${ }^{3}$ G. Abrasonis, ${ }^{3}$ J. Fassbender, ${ }^{3}$ M. D. Baró, ${ }^{4}$ J. Sort, ${ }^{5}$ \\ and J. Nogués ${ }^{6}$ \\ ${ }^{1}$ Instituut voor Kern- en Stralingsfysica and INPAC, Katholieke Universiteit Leuven, Celestijnenlaan 200 D, \\ BE-3001 Leuven, Belgium \\ ${ }^{2}$ Institut PPRIME UPR3346 CNRS, ENSMA, Université de Poitiers, Futuroscope-Chasseneuil, France \\ ${ }^{3}$ Institute of Ion Beam Physics and Materials Research, Forschungszentrum Dresden-Rossendorf, \\ P.O. Box 510119, D-01314 Dresden, Germany \\ ${ }^{4}$ Departament de Física, Univ. Autònoma de Barcelona, E-08193 Bellaterra, Spain \\ ${ }^{5}$ Departament de Física and Institució Catalana de Recerca i Estudis Avançats (ICREA), Univ. Autònoma \\ de Barcelona, E-08193 Bellaterra, Spain \\ ${ }^{6}$ Institució Catalana de Recerca $i$ Estudis Avançats (ICREA) and Centre d'Investigació en Nanociència $i$ \\ Nanotecnologia (ICN-CSIC), Campus Univ. Autònoma de Barcelona, E-08193 Bellaterra, Spain
}

(Received 7 April 2010; accepted 20 May 2010; published online 18 June 2010)

\begin{abstract}
A correlation between the grain orientation and the out-of-plane magnetic properties of nitrogen-enriched polycrystalline austenitic stainless steel surface is performed. Due to the competition between the magnetocrystalline anisotropy, the exchange and dipolar interactions, and the residual stresses induced by nitriding, the resulting effective magnetic easy-axis can lay along unusual directions. It is also demonstrated that, by choosing an appropriate stainless steel texturing, arrays of ferromagnetic structures with out-of-plane magnetization, embedded in a paramagnetic matrix, can be produced by local plasma nitriding through shadow masks. () 2010 American Institute of Physics. [doi:10.1063/1.3453567]
\end{abstract}

Developments in stainless steels are leading to functionalities which are progressively broadening their applications into more advanced end products (like force transducers or impellers for centrifugal compressors). ${ }^{1}$ Diverse surface treatments are employed to enhance the properties of austenitic stainless steels (ASSs), which often result in the formation of other phases, such as martensite (rolling) ${ }^{2}$ or expanded austenite after nitriding at moderate temperatures. ${ }^{3-5}$ The expanded austenite exhibits improved hardness, ${ }^{6}$ wear resistance, ${ }^{7}$ and fatigue resistance. ${ }^{8,9}$ Remarkably, both of these induced phases are ferromagnetic at room temperature, in contrast to ASSs which are paramagnetic. ${ }^{10-12}$ Nevertheless, relatively little is known about the magnetic properties of the expanded austenite. Both mechanical ${ }^{13,14}$ and ion beam nitriding ${ }^{15}$ have been exploited to locally generate ferromagnetic structures on ASSs (i.e., magnetic patterning). However, only in-plane magnetization has been demonstrated up-to-date in these magnetic patterning methods. Notably, for many applications (e.g., magnetic recording), ${ }^{16}$ outof-plane magnetization would be favorable.

A mirror-polished rectangular specimen $22 \times 18 \mathrm{~mm}^{2}$ of 316L-type polycrystalline ASS has been plasma-nitrided at $400{ }^{\circ} \mathrm{C}$ for $30 \mathrm{~min} .{ }^{17,18}$ This results in $\mathrm{a} \approx 0.8 \mu \mathrm{m}$ thick nitrided layer with a concentration of nitrogen in the first $0.1 \mu \mathrm{m}$ of about 25 at. $\%{ }^{19}$ For the magnetic patterning, a $5 \mu \mathrm{m}$ thick and $3.05 \mathrm{~mm}$ in diameter 1500 mesh $\mathrm{Cu}$ transmission electron microscopy (TEM) grid (with 11.5 $\times 11.5 \mu \mathrm{m}^{2}$ square holes in a $16.5 \mu \mathrm{m}$ pitch) is placed as a mask at the sample surface and it is mechanically held by an especially designed Ti holder with a rectangular-shaped hole with wedged edges to minimize shadowing effects. The crys-

${ }^{a)}$ Electronic mail: enric.menendezdalmau@ fys.kuleuven.be. tallographic orientations of the grains after nitriding have been determined by means of electron backscatter diffraction (EBSD) on a scanning electron microscope JEOL 6100, equipped with an orientation imaging microscopy system. The probed depth in EBSD measurements is typically $0.1 \mu \mathrm{m}$ and the uncertainty on the orientation is around $0.5^{\circ}{ }^{20}$ Determination of the crystallographic orientations from EBSD patterns has been performed considering a facecentered cubic (fcc) structure since the nitrided regions retain the structure of the host austenite ${ }^{21}$ with an expanded lattice parameter. ${ }^{22}$ Atomic force microscopy /magnetic force microscopy (AFM/MFM, Veeco/DI Dimension 3100) imaging has been used to examine the surface topography and the local magnetic domain structure, respectively. Note that the MFM images have been recorded in samples not previously exposed to any magnetic fields). The magnetic properties of the homogeneously nitrided areas and the patterned regions have been determined by polar magneto-optical Kerr effect (MOKE) magnetometry (Durham Magneto-optics). Namely, the direction of the applied magnetic field coincides with the normal direction (ND) to the sample surface (i.e., out-ofplane). Since the employed laser spot of the MOKE apparatus has been focused down to a diameter of $5 \mu \mathrm{m}$ and the investigated samples have an average grain size of $50 \mu \mathrm{m}$, the magnetic behavior of individual grains has been determined.

The virgin ASS 316L grains do not show any ferromagnetic signal, confirming their paramagnetic character. After nitriding, they become ferromagnetic which is related to the formation of expanded austenite. This is demonstrated in Fig. 1 gathering the magnetic response of four representative investigated grains. The magnetic response of the sample depends distinctly on the measuring location, i.e., on the inves- 
(a)

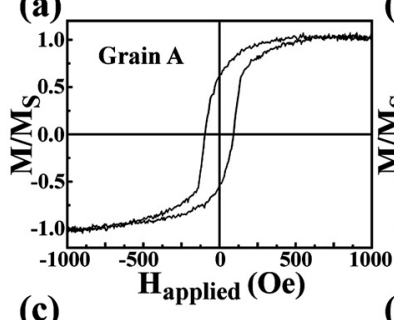

(b)

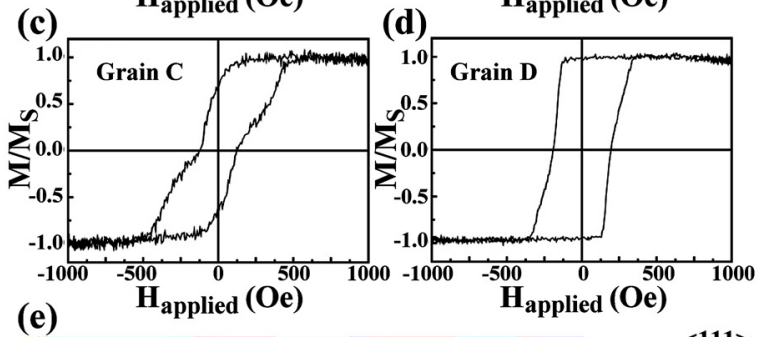

(e)

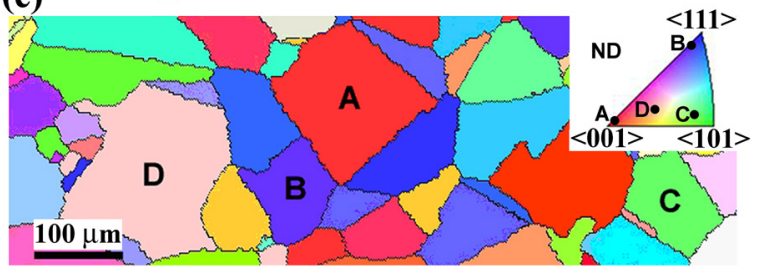

FIG. 1. (Color online) (a), (b), (c), and (d) individual polar MOKE measurements of grains A, B, C, and D, respectively. (e) EBSD map of the investigated area showing the crystalline orientation of the grains according to the ND of the sample surface. The color code is presented by the associated inverse pole figure, see inset of panel (e). M, $\mathrm{M}_{\mathrm{S}}$, and $\mathrm{H}_{\text {applied }}$ stand for magnetization, saturation magnetization and applied magnetic field, respectively.

tigated grain. Although nitrogen concentration profile depends on crystalline orientation, the differences are small close to the surface. ${ }^{23}$ Since MOKE probes the first tens of nanometers, the effect of the nitrogen concentration on the obtained magnetic results is minimized. A large variety of behaviors is observed, such as a sheared shape loop with relatively small coercivity $\left(\mathrm{H}_{\mathrm{C}}\right)$ [Fig. 1(a), grain $\mathrm{A}$ ], squarelike loops with a high remanence and a moderate $\mathrm{H}_{\mathrm{C}}$ [Fig. 1(d), grain D] or unusual loop shapes [Figs. 1(b) and 1(c), grains $\mathrm{B}$ and $\mathrm{C}]$. These indicate that the ND to the sample surface of the grains [see Fig. 1(e)] is oriented differently with respect to the magnetic easy-axes. Therefore, while the orientation of grain D would be close to an easy-axis, grain A would have a more hard-axis behavior. Unfortunately, the easy-axis directions for expanded austenite are unknown. This fact together with the concomitant exchange and dipolar coupling between grains and the plastic deformation induced by nitriding ${ }^{24-26}$ make the analysis of the loops rather complex. Nevertheless, the stepped loops for grains B and C may arise from the presence of two competing anisotropies, e.g., cubic (perhaps intrinsic to the expanded austenite) and uniaxial (e.g., due to residual strain) and a measuring field away from the easy-axes. ${ }^{27,28}$ The crystalline orientation of grain $\mathrm{A}$ is close to $\langle 001\rangle$, and relatively close to $\langle 111\rangle$, and $\langle 101\rangle$ for the grains B and C. The orientation of grain D $(\approx\langle 214\rangle)$ is far from the directions of low indices. Thus, the magnetic and structural measurements indicate that the effective easy-axis is not along the $\langle 111\rangle$ direction, as expected for fcc materials, ${ }^{29}$ but lies along unusual directions as a result of interactions and multiple easy-axes.

Concerning the patterned areas, a regular array of 11.5 $\times 11.5 \mu \mathrm{m}^{2}$ square-shaped ferromagnetic structures embed- (a)

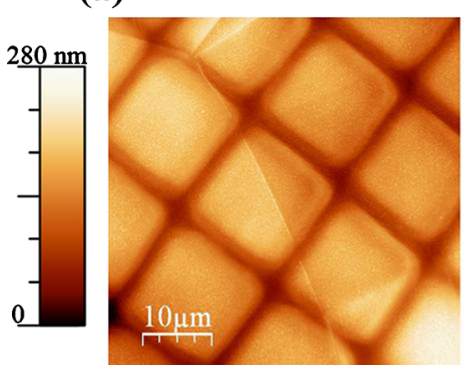

(b)

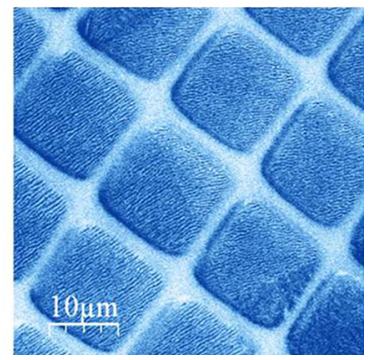

FIG. 2. (Color online) Surface topography (a) and magnetic domain configuration (b), determined by AFM and MFM, of an ASS surface area nitrided through the mask. The scale bar represents the surface height.

ded in a paramagnetic matrix is generated (see Fig. 2). Note that grains with diverse crystallographic orientations have been patterned and, similarly to the homogeneously nitrided case, the magnetic response varies from grain to grain [Fig. 2(b)]. Since the surface sputtering is virtually negligible during the plasma nitriding at the floating potential, the topography is due to the swelling phenomenon induced by the nitrogen incorporation with an average value of $\approx 200 \mathrm{~nm}$. In the patterned region, the non-nitrided areas should be also slightly expanded due to the lateral diffusion of nitrogen. Nonetheless, as it can be seen in the MFM image of Fig. 2, the lateral diffusion does not enlarge sufficiently the lattice cell to make it become ferromagnetic. The lack of MFM contrast between the entities demonstrates the confinement of the magnetic patterning. In the patterned area, the correlation between the magnetic behavior [Figs. 3(a)-3(c)] and the crystallographic orientations [Fig. 3(d)] is similar to the one observed in the homogeneously nitrided region. Namely,

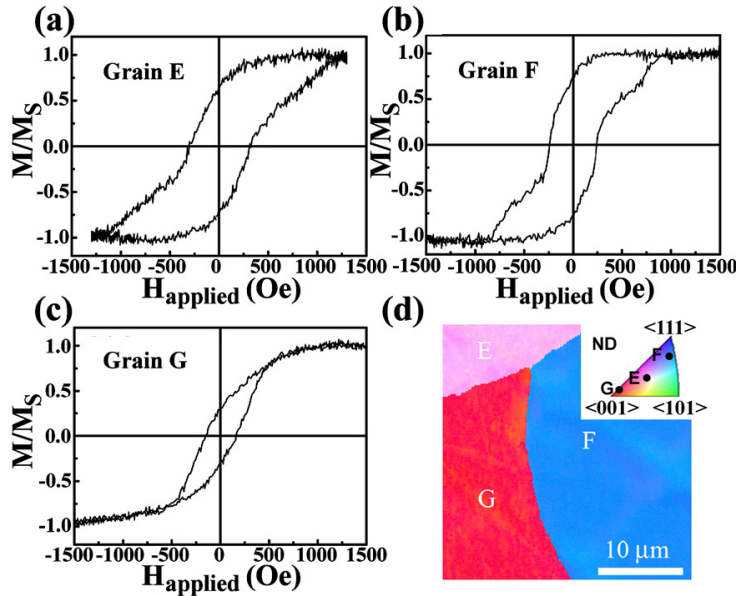

(e)

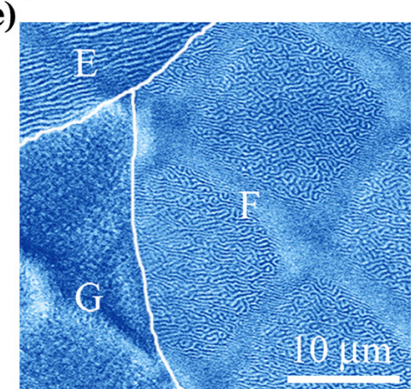

FIG. 3. (Color online) (a), (b), and (c) individual polar MOKE measurements of grains E, F, and G, respectively. (d) EBSD map of this area which shows the crystalline orientation of the grains according to the ND of the sample surface with an inserted ND inverse pole figure presenting the color code. (e) MFM image of the investigated area. 
while grain $\mathrm{G}$ (close to $\langle 001\rangle$ ) exhibits a hard-axis behavior [Fig. 3(c)], grains E (orientation close to that of grain D) and $\mathrm{F}$ (close to $\langle 111\rangle$ ) show a somewhat high remanent magnetization and a rather large coercivity, see Figs. 3(a) and 3(b), implying that their orientations are closer to that of an easyaxis. Further evidence for the correlation between crystalline orientation and magnetic response can be found from the MFM image [Fig. 3(e)]. Grain G shows no clear magnetic contrast, probably indicating that the magnetization lies inplane and no domain wall can be observed within the field of view. This is compatible with the hard-axis behavior of the polar-loop of this grain [see Fig. 3(c)]. Grains E and F both exhibit stripped magnetic domains typical of out-of-plane magnetization. However, the periodicity and the morphology of the domains are quite different (note that, while grain $\mathrm{E}$ shows stripe domains, grain $\mathrm{F}$ evidences labyrinth-shaped ones). The different domain morphologies result mainly from the balance between exchange, anisotropy, and dipolar energies and are highly dependent on the magnetic field history. ${ }^{30}$ Thus, the change in domain morphology in our case is probably due to the different degree of alignment of these grains with the effective easy-axis. In contrast to the patterned area of Fig. 2(b), the ferromagnetic entities shown in Fig. 3(e) are slightly interconnected possibly due to a misalignment (e.g., poor mask-sample contact) of the TEM grid with the sample surface at this location. Interestingly, in the patterned sample, the fields necessary to saturate the magnetization and the coercivities are larger compared to those of the homogeneously nitrided sample (e.g., $\mathrm{H}_{\mathrm{C}}=181$ Oe for grain B-homogeneous area and $\mathrm{H}_{\mathrm{C}}=242 \mathrm{Oe}$ for grain F-patterned area). This is expected since the edges of the patterns can pin the domain walls making the magnetization reversal by domain wall movement more difficult. ${ }^{31}$ Furthermore, the change in the crystallographic orientation due to nitriding, ${ }^{32}$ clearly observed in Fig. 3(d), renders a even more complicated interplay between the domain walls and these edges.

In summary, magnetic properties of individual grains of homogeneously and patterned plasma-nitrided polycrystalline ASS 316L have been investigated by means of EBSD, MOKE, and AFM/MFM. The magnetic response of grains is correlated to the crystallographic orientation. Grains oriented close to $\langle 001\rangle$ exhibit mainly in-plane magnetization while grains with orientations close to $\langle 111\rangle$ or $\langle 101\rangle$ have a more pronounced out-of-plane response. Remarkably, the resulting effective magnetic easy-axis is not $\langle 111\rangle$, as expected for fcc materials, but an unusual direction. This could be due to the interplay between the magnetocrystalline anisotropy, the exchange and dipolar interactions between the grains, and the residual stresses induced by the nitriding process. In addition, it is shown that by an appropriate choice of the initial stainless steel texturing in combination with nitriding through shadow masks can be used to fabricate patterned magnetic materials with out-of-plane magnetization. This could pave the way for a potential simple method to fabricate magnetic devices.
This work has been partially financed by the Catalan DGR and the Spanish MICINN (Grant Nos. 2009-SGR1292, MAT2007-66309-C02, and MAT2007-61629). E.M. thanks the Fund for Scientific Research-Flanders FWO for financial support. M.D.B. was partially supported by an ICREA ACADEMIA award.

${ }^{1}$ K. H. Lo, C. H. Shek, and J. K. L. Lai, Mater. Sci. Eng. R. 65, 39 (2009).

${ }^{2}$ V. Shrinivas, S. K. Varma, and L. E. Murr, Metall. Mater. Trans. A 26, 661 (1995).

${ }^{3}$ G. A. Collins, R. Hutchings, K. T. Short, J. Tendys, X. Li, and M Samandi, Surf. Coat. Technol. 74-75, 417 (1995).

${ }^{4}$ T. Czerwiec, H. He, G. Marcos, T. Thiriet, S. Weber, and H. Michel, Plasma Processes Polym. 6, 401 (2009).

${ }^{5}$ T. Christiansen and M. Somers, Surf. Eng. 21, 445 (2005).

${ }^{6}$ E. Richter, R. Günzel, S. Parascandola, T. Telbizova, O. Kruse, and W. Möller, Surf. Coat. Technol. 128-129, 21 (2000).

${ }^{7}$ T. Bell, Surf. Eng. 18, 415 (2002).

${ }^{8}$ K. Genel, M. Demirkol, and M. Çapa, Mater. Sci. Eng., A 279, 207 (2000).

${ }^{9}$ S. Sirin, K. Sirin, and E. Kaluc, Mater. Charact. 59, 351 (2008).

${ }^{10}$ S. S. Hecker, M. G. Stout, K. P. Staudhammer, and J. L. Smith, Metall. Trans. A 13, 619 (1982).

${ }^{11}$ E. Menéndez, J. Sort, M. O. Liedke, J. Fassbender, S. Suriñach, M. D. Baró, and J. Nogués, J. Mater. Res. 24, 565 (2009).

${ }^{12}$ O. Öztürk and D. L. Williamson, J. Appl. Phys. 77, 3839 (1995).

${ }^{13}$ J. Sort, A. Concustell, E. Menéndez, S. Suriñach, M. D. Baró, J. Farran, and J. Nogués, Appl. Phys. Lett. 89, 032509 (2006).

${ }^{14}$ H. Ishigaki, Y. Konishi, K. Kondo, and K. Koterazawa, J. Magn. Magn. Mater. 193, 466 (1999).

${ }^{15}$ E. Menéndez, A. Martinavicius, M. O. Liedke, G. Abrasonis, J. Fassbender, J. Sommerlatte, K. Nielsch, S. Suriñach, M. D. Baró, J. Nogués, and J. Sort, Acta Mater. 56, 4570 (2008).

${ }^{16}$ H. N. Bertram and M. Williams, IEEE Trans. Magn. 36, 4 (2000).

${ }^{17}$ J. Perrière, J. Siejka, N. Rémili, A. Laurent, A. Straboni, and B. Vuillermoz, J. Appl. Phys. 59, 2752 (1986).

${ }^{18}$ L. Marot, A. Straboni, and M. Drouet, Surf. Coat. Technol. 142-144, 384 (2001)

${ }^{19}$ C. Templier, J. C. Stinville, P. Villechaise, P. Renault, G. Abrasonis, J. P. Rivière, A. Martinavičius, and M. Drouet, Surf. Coat. Technol. 204, 2551 (2010)

${ }^{20}$ D. Dingley and V. Randle, J. Mater. Sci. 27, 4545 (1992).

${ }^{21}$ M. P. Fewell and J. M. Priest, Surf. Coat. Technol. 202, 1802 (2008).

${ }^{22}$ T. Christiansen and M. A. J. Somers, Metall. Mater. Trans. A 37, 675 (2006).

${ }^{23}$ A. Martinavičius, G. Abrasonis, W. Möller, C. Templier, J. P. Rivière, A. Declémy, and Y. Chumlyakov, J. Appl. Phys. 105, 093502 (2009).

${ }^{24}$ S. Grigull and S. Parascandola, J. Appl. Phys. 88, 6925 (2000).

${ }^{25}$ X. Xu, Z. Yu, L. Wang, J. Qiang, and Z. Hei, Surf. Coat. Technol. 162, 242 (2003).

${ }^{26}$ J. C. Stinville, P. Villechaise, C. Templier, J. P. Rivière, and M. Drouet, Surf. Coat. Technol. 204, 1947 (2010)

${ }^{27}$ Q. F. Zhan, S. Vandezande, K. Temst, and C. Van Haesendonck, Phys. Rev. B 80, 094416 (2009).

${ }^{28}$ J. L. Costa-Krämer, J. L. Menéndez, A. Cebollada, F. Briones, D. García and A. Hernando, J. Magn. Magn. Mater. 210, 341 (2000).

${ }^{29}$ S. Chikazumi, Physics of Magnetism (Wiley, New York, 1964).

${ }^{30}$ O. Hellwig, A. Berger, J. Kortright, and E. E. Fullerton, J. Magn. Magn. Mater. 319, 13 (2007).

${ }^{31}$ J. I. Martín, J. Nogués, K. Liu, J. L. Vicent, and I. K. Schuller, J. Magn Magn. Mater. 256, 449 (2003).

${ }^{32}$ J. C. Stinville, P. Villechaise, C. Templier, J. P. Rivière, and M. Drouet, Acta Mater. 58, 2814 (2010) 